\title{
Sur la cristallisation xénobiologique de l'hémoglobine sanguine des Souris et des Rats blancs de laboratoire normaux et infestés par le Trypanosoma cruzi le Plasmodium bergbei, ou par différentes souches du virus grippal
}

\author{
Par F. PICK
}

Les cristaux de l'hèmoglobine sanguine de la Souris ont été observés, pour la première fois, en 1853, par F. Kunde (5), qui a signalé avoir obtenu des aiguilles «parfaites » en ajoutant simplement de l'eau au sang à examiner et, après addition d'éther, des plaques prismatiques qui ont disparu 10 minutes après leur élaboration.

Dans la même note, Kunde mentionne que le sang du Rat cristallise, d'après une communication personnelle de Lehmann, sous forme de tétraèdres comme le sang du Cobaye.

Lehmann (6), pour sa part, avait communiqué en 1852: «J'ai observé que le sang de Souris (comme celui de Cobaye), de même que Kunde pour le sang du Rat, cristallisait sous forme tétraédrique. »

La grande autorité de Lehmann sur le plan de la physiologie avait empêché de contester la réalité des observations sus-mentionnées.

Ce n'est qu'en 1863 que nous rencontrons une description plus précise des cristaux du sang de la Souris donnée par C. Bojanowski (1).

La technique de cet auteur pour la préparation des cristaux sanguins a consisté dans l'abandon du sang prélevé ou laissé dans le cadavre pendant deux à quatre jours, dans un endroit un peu froid. Si la liquéfaction du sang coagulé n'était pas suffisante, un peu d'eau était ajoutée. La préparation effectuée entre lame et lamelle était exposée à la lumière pendant quelques heures. Dans ces conditions, Bojanowski a pu observer que le sang de la Souris donnait des cristaux d'hématocrystalline, sous forme de plaques hexagonales régulières. 


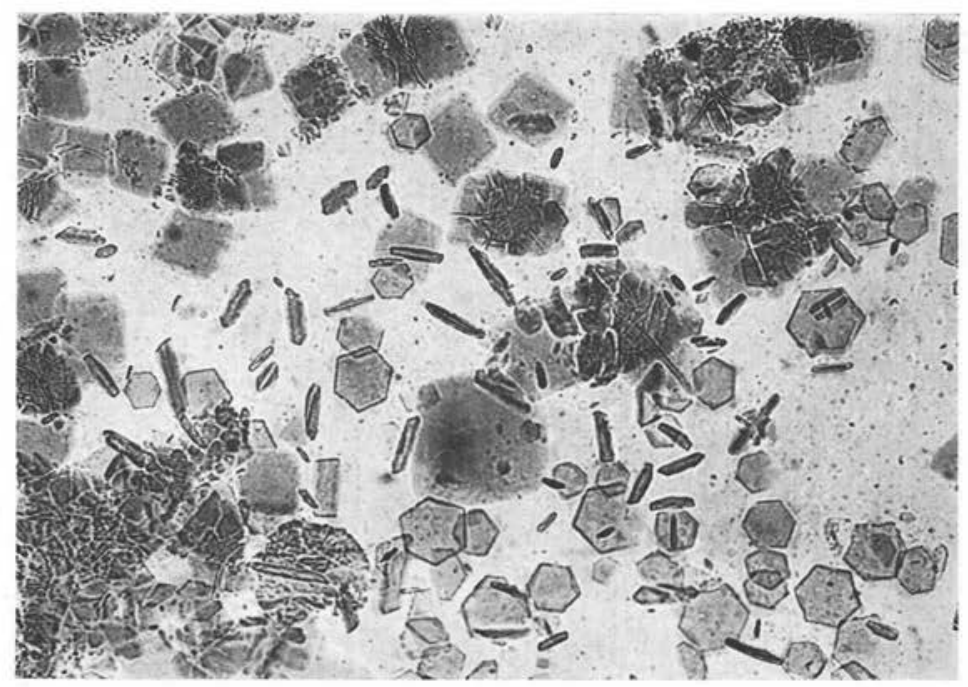

Fig. 1. - Document montrant la présence simultanée de cristaux d'hémoglobine élaborés au cours de la phase biologique directe (plaques hexagonales régulières et bâtonnets) et de cristaux d'hémoglobine élaborés au cours de la phase biologique indirecte (formes quadrangulaires). La diagonale du grand cristal quadrangulaire du milieu de la figure est de $400 \mu$

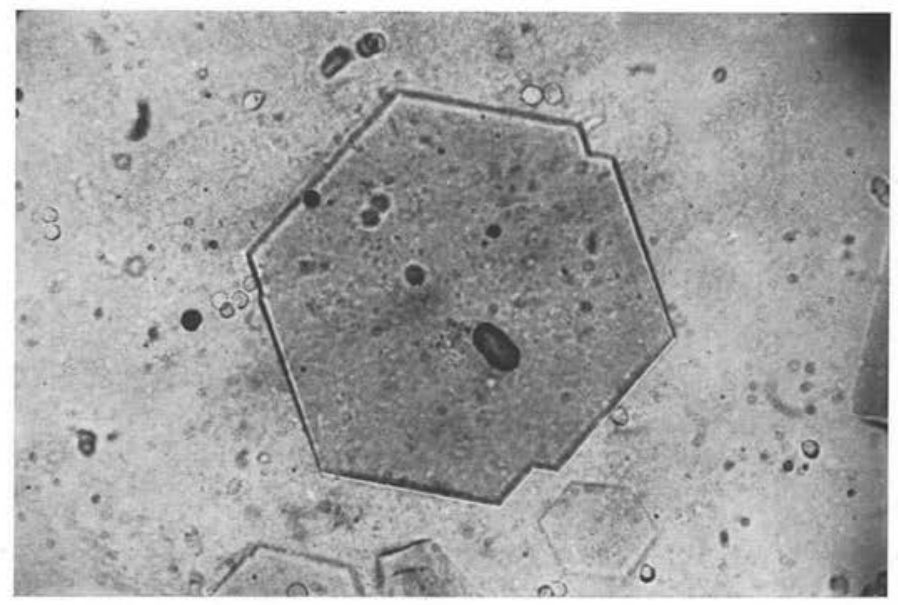

FIG. 2. - Cristallisation xénobiologique de l'hémoglobine sanguine de la Souris par Triatoma infestans, montrant une forme hexagonale à incisions périphériques. Le diamètre transversal de ce cristal est de $168 \mu$ 
En 1887, C. J. Bond (2) n'a signalé que la facilité avec laquelle le sang du Rat, de la Souris sauvage et domestique ( tame mouse $»)$ cristallise en 20 à 30 heures, entre lame et lamelle, dans les hématies et dans le sérum.

En 1890, S. Monckton Copeman (7) a mentionné l'obtention des cristaux du Rat et de la Souris, à partir de leur sang traité par l'éther, sans autre description précise.

Ch. Richet (12), en donnant un aperçu histcrique des travaux portant sur les formes cristallines de l'oxyhémoglobine, a rappelé que celles du Rat se présentent sous forme d'octaèdres ou de tétraèdres.

C'était dans la même année que E. T. Reichert et A. P. Brown (11) ont publié leur grande monographie sur la cristallographie de l'hémoglobine.

Ces auteurs ont pu examiner le sang de quatre espèces de Rats (blanc, brun, noir et alexandrin), qui ont montré de légères différences dans leur cristallisation dans le système orthorhombique commun. Il s'agissait de plaques prismatiques allongées hexagonales.

En 1926, H. Goffart (4) a préparé des cristaux d'oxyhémoglobine d'un Rat (Mus decumanus Pall.) par voie physico-chimique, reproduisant des dessins sous forme de prismes hexagonaux allongés, hexagonaux réguliers, ou terminés par des triangles, et des trapèzes.

Dans l'anémie des Rats, après splénectomie, E. Sorina (13) a constaté que le sang de ces Rats cristallise spontanément entre lame et lamelle. Le document microphotographique de Sorina, pris dans le champ obscur, montre des cristaux hexagonaux allongés.

En 1930 enfin, A. K. Boor (3) en utilisant une technique physico-chimique, différente de celle employée par Reichert et Brown (11), pour le sang du Rat sauvage et croisé, a constaté que les cristaux d'oxyhémoglobine et de carboxyhémoglobine du sang de ces animaux paraissent être des plaques hexagonales allongées.

En présence des réponses cristallographiques sus-mentionnées, nous nous sommes adressés aux Réduvidés hématophages pour faire cristalliser l'hémoglobine sanguine du Rat albinos et de la Souris blanche par voie biologique directe et indirecte, en nous proposant en même temps d'examiner la répercussion éventuelle d'une infestation par le $T$. cruzi ou le $P$. berghei, ainsi aue d'une infection par différents virus de la grippe sur les cristallisations réduvidiques.

Ainsi, nous avons pu constater que l'hémoglobine du Rat albinos cristallise au niveau de l'estomac des Réduvidés hématophages sous forme de plaques hexagonales symétriques d'une part et sous forme de bâtonnets d'autre part (fig. 1).

$\mathrm{Au}$ cours de la cristallisation biologique continue, entre lame et lamelle, ou « cristallisation biologique indirecte », ou « cristallisation post-réduvique », ou encore « cristallisation xénobiologique indirecte », nous avons observé l'apparition de cristaux d'hémoglobine plus ou moins quadrangulaires (fig. 1).

Le sang de la Souris blanche de laboratoire, soumise aux mêmes réactions biologiques, conduit par voie directe à l'élaboration de formes hexagonales dont la régularité est interrompue par des incisions géométriques périphériques et des cristaux en forme de bâtonnets (fig. 2 et 3 ). 


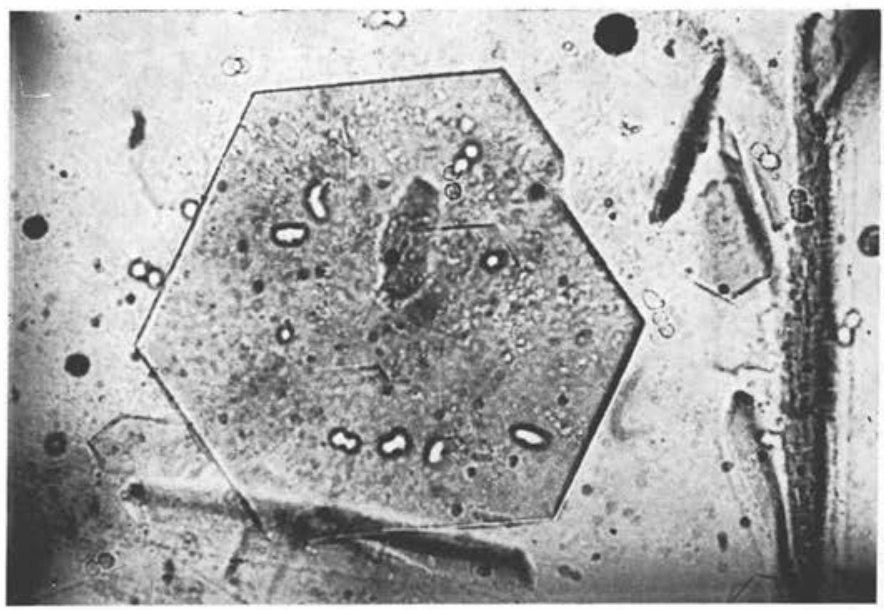

FIG. 3. - Cristallisation xénobiologique directe et indirecte de l'hémoglobine de la Souris par $T$. infestans montrant un cristal hexagonal imparfait d'un diamètre transversal de $196 \mu$ et des cristaux en forme de gros bâtonnets

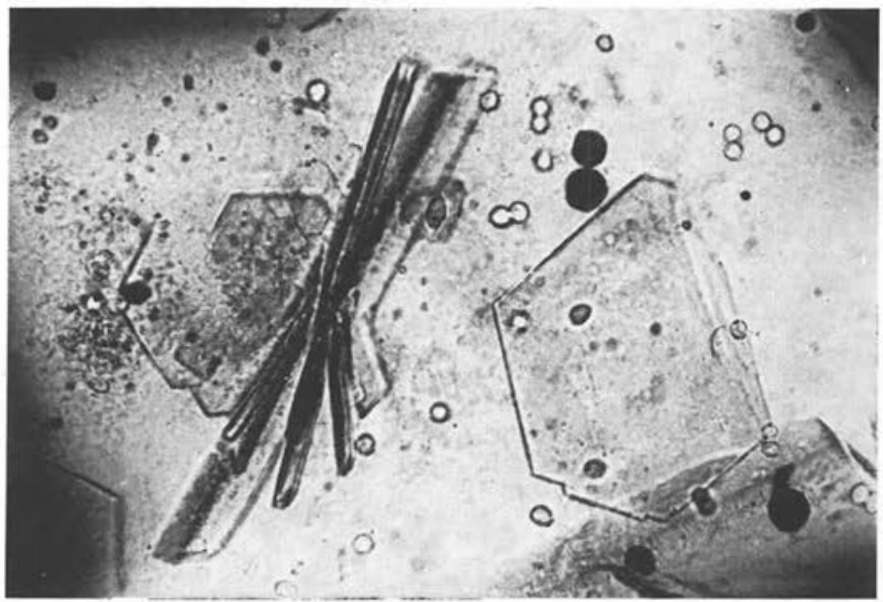

FIG. 4. - Cristallisation post-réduvidique de l'hémoglobine sanguine de la Souris, montrant un faisceau de grands cristaux prismatiques très allongés, qui a une extension longitudinale de $225 \mu$. Le cristal hexagonal résiduel montre, dans la partie inférieure de sa délimitation linéaire persistante, deux incisions 
La cristallisation post-réduvidique de l'hémoglobine sanguine de la Souris montre surtout une notable croissance des formes en bâtonnets, tandis que l'élaboration de cristaux d'aspect quadrangulaire est sensiblement moins prononcée que celle de la phase correspondante chez le Rat.

Cette croissance post-réduvidique des cristaux d'hémoglobine, se trouvant initialement sous forme de bâtonnets mal délimités, peut les transformer en cristaux nettement prismatiques (fig. 4), qui ne s'observent pas chez le Rat albinos.

Dans des notes antérieures $(8,9)$, nous avons signalé que le Trypanosoma cruzi n'intervient pas dans le processus des cristallisations biologiques en général.

Il n'y avait donc rien d'étonnant à constater qu'il n'y avait pas non plus de répercussion chez le Rat adulte qui ne fait que des infestations passagères, de même que chez la Souris qui fait des infestations mortelles par le $T$. cruzi.

Par contre, des expériences effectuées en collaboration avec L. Lamy, portant sur la répercussion éventuelle d'une infestation de ces Muridés par le Plasmodium berghei (souche de H. Galliard), nous ont révélé une intervention appréciable de ce parasite sanguicole dans les cristallisations biologiques de l'hémoglobine, surtout dans les cas de la Souris blanche.

Nous basant sur des essais ultérieurs (10), portant sur la répercussion du Plasmodium vivax dans des cristallisations spontanées de l'hémoglobine sanguine de psychopathes, nous pensons que, chez $P$. berghei, ce sont également des produits toxiques qui sont responsables de l'intervention dans les cristallisations biologiques des Muridés examinés.

Enfin, nous avons procédé à des expériences portant sur la répercussion éventuelle de l'infection du Rat albinos par différentes souches de grippe, mises à notre disposition par le Service de M. Dujarric de la Rivière,, sur les cristallisations biologiques de ces Rongeurs.

Dans ces cas aussi, nous avons pu constater des perturbations appréciables dans les cristallisations biologiques de l'hémoglobine du Rat.

Nous reviendrons ultérieurement, avec plus de détail, sur les perturbations causées par le $P$. berghei et le virus de la grippe sur les crisallisations biologiques de l'hémoglobine.

\section{En Résumé}

Par la technique de xénodiagnostic biologique par l'intermédiaire des Réduvidés hématophages, nous avons pu constater que :

$1^{\circ}$ l'hémoglobine sanguine du Rat albinos cristallise directement sous forme de plaques hexagonales régulières et de bâtonnets, et, indirectement, sous forme de cristaux quadrangulaires ;

$2^{\circ}$ l'hémoglobine sanguine de la Souris blanche cristallise directement sous forme de plaques hexagonales dont la délimitation linéaire est interrompue par quelques incisions, et, indirectement, par la croissance des formes en bâtonnets devenant prismatiques ; 
$3^{\circ}$ le $T$. cruzi n'intervient pas dans les cristallisations biologiques de l'hémoglo. bine des Muridés examinés ;

$4^{\circ}$ le $P$. berghei et différentes souches du virus de la grippe interviennent d'une manière appréciable dans les cristallisations biologiques de l'hémoglobine sanguine du Rat albinos, fournissant des réponses cristallographiques sur lesquelles nous reviendrons ultérieurement.

\section{Bibliographie}

1. BojanowsKi (C.), 1863. - Beobachtungen über Blutkrsytalle. Ztschr. wiss. Zoologie, XII, 312-335.

2. Bond (C. J.), 1887. - A contribution to the pathology of the blood. Lancet., II, 509-511 et $557-560$.

3. Boor (A. K.), 1930. - A crystallographic study of pure carbonmonoxide hemoglobin. J. Gen. Physiol., XIII, 307-316.

4. Goffart (H.), 1926. - Beitrag zur Kenntnis der Oxyhämoglobinkristalle. Zool. Jahrb., Abt. Allg. Zool. u. Physiol., XLII, 193-242.

5. Kunde (F.), 1852. - Ueber Krystallbildung im Blute. Ztschr. rat. Med., II, 271-287.

6. Lehmann, 1852. - Ueber die Krystallisirbarkeit eines der Hauptbestandtheile der Blutkörperchen. Ber. Verdln. Kgl. sächs. Ges. Wiss. Math.-Phys. Cl., 23-26.

7. Monckton Copeman (S.), 1890. - The crystallization of haemoglobin in Man and the lower animals, and of haemochromogen in Man. Jl. Physiology, XI, 401-409.

8. PICK (F.), 1951. - Sobre la cristalizaciòn de la sangre ingerída por triatomas. Nueva reacciòn biològica en los redùvidos hematòfagos. Arch. Soc. Biol. Montevideo, $X V I I I, 100-103$.

9. PICK (F.), 1952. - Sur la cristallisation biologique du sang ingéré par Réduvidés du genre Triatoma. Bull. Soc. Path. exot., XL, 326-328.

10. Pick (F.), 1959. - Répercussion de l'impaludation de psychopathes sur la cristallisation spontanée de l'hémoglobine in vitro du sang frais. Bull. Soc. Path. exot., LIV, 12091212.

11. Reichert (E. T.) et Brown (A. P.), 1909. - The crystallography of hemoglobin. Carnegie Inst. Washington, Publ. $\mathrm{n}^{\circ} 116, \mathrm{xIX}+338 \mathrm{pp}$.

12. Richet (Ch.), 1909. - Dictionnaire de Physiologie, Paris, 1909.

13. Sorina (E.), 1928. - Anämie der Ratten nach Entmilzung. Arch. Pathol. Anat. Physiol. u. klin. Med., CCLXX, 698-705.

( $P^{r}$ François Pick, Box 51, Vienve 71, Autriche) 